1. Antic NA, Heeley E, Anderson CS, et al. The Sleep Apnea cardioVascular Endpoints (SAVE) trial: rationale, ethics, design, and progress. Sleep 2015;38:1247-57.

2. Weaver TE, Grunstein RR. Adherence to continuous positive airway pressure therapy: the challenge to effective treatment. Proc Am Thorac Soc 2008;5:173-8.

3. Kritikou I, Basta M, Tappouni R, et al. Sleep apnoea and visceral adiposity in middle-aged male and female subjects. Eur Respir J 2013;41:601-9.
4. Kim AM, Keenan BT, Jackson N, et al. Tongue fat and its relationship to obstructive sleep apnea. Sleep 2014;37:163948.

5. Woodward M, Patel A, Zoungas S, et al. Does glycemic control offer similar benefits among patients with diabetes in different regions of the world? Results from the ADVANCE trial. Diabetes Care 2011;34:2491-5.

DOI: $10.1056 / \mathrm{NEJMC1613219}$

\title{
Variants in ANGPTL4 and the Risk of Coronary Artery Disease
}

TO THE EDITOR: Genomic studies of cardiovascular disease that are based on samples obtained from patients have identified variants associated with the risk of disease that do not always predict the onset of disease shown in prospective studies. ${ }^{1,2}$ Dewey et al. (March 24 issue) ${ }^{3}$ report lower levels of triglycerides and a lower risk of coronary artery disease among carriers of $\mathrm{E} 40 \mathrm{~K}$ and other inactivating mutations in ANGPTL4 than among noncarriers. Also, in the same issue of the Journal, Stitziel et al. report that in a cross-sectional study, low-frequency coding-sequence variants were associated with a reduced risk of coronary disease. $^{4}$

We carried out an independent, large-scale study and confirmed the role of these genetic variants in incident coronary heart disease. Our study involved data that was collected prospectively from seven cohorts (the Age, Gene/Environment Susceptibility-Reykjavik Study; the Atherosclerosis Risk in Communities Study; the Cardiovascular Health Study; the Framingham Heart Study; the Genetic Studies of Atherosclerosis Risk; the Rotterdam Study; and the Women's Genome Health Study). A total of 5427 of 49,518 persons of European ancestry in these studies had incident coronary heart disease. ${ }^{5}$

We investigated 35,904 autosomal exome-array variants with a minor-allele frequency of greater than $1 \%$ for association, with adjustment for age, sex, and population substructure. Significant associations (Bonferroni-corrected $\mathrm{P}<1.39 \mathrm{E}-06$ ) are summarized in Table S1 in the Supplementary Appendix, available with the full text of this letter at NEJM.org. We confirm previously reported $^{6}$ associations with noncoding common variants at 9p21 and PHACTR1, and we also found an association between a low-frequency variant in ANGPTL4 and a reduced risk of incident coronary heart disease.

\author{
Alanna C. Morrison, Ph.D. \\ University of Texas Health Science Center at Houston \\ Houston, TX \\ alanna.c.morrison@uth.tmc.edu \\ Yi-Ping Fu, Ph.D. \\ National Heart, Lung, and Blood Institute \\ Bethesda, MD
}

Christopher J. O'Donnell, M.D., M.P.H.

Boston Veterans Administration Healthcare System Boston, MA

for the Cohorts for Heart and Aging Research in Genomic Epidemiology (CHARGE) Consortium Subclinical Atherosclerosis and CHD Working Group

No potential conflict of interest relevant to this letter was reported.

1. Dehghan A, Bis JC, White CC, et al. Genome-wide association study for incident myocardial infarction and coronary heart disease in prospective cohort studies: the CHARGE Consortium. PLoS One 2016;11(3):e0144997.

2. Franceschini N, Hu Y, Reiner AP, et al. Prospective associations of coronary heart disease loci in African Americans using the MetaboChip: the PAGE study. PLoS One 2014;9(12):e113203. 3. Dewey FE, Gusarova V, O'Dushlaine C, et al. Inactivating variants in ANGPTL4 and risk of coronary artery disease. N Engl J Med 2016;374:1123-33.

4. Myocardial Infarction Genetics and CARDIoGRAM Exome Consortia Investigators. Coding variation in ANGPTL4, LPL, and SVEP1 and the risk of coronary disease. N Engl J Med 2016;374: 1134-44.

5. Grove ML, Yu B, Cochran BJ, et al. Best practices and joint calling of the HumanExome BeadChip: the CHARGE Consortium. PLoS One 2013;8(7):e68095.

6. Nikpay M, Goel A, Won HH, et al. A comprehensive 1,000 Genomes-based genome-wide association meta-analysis of coronary artery disease. Nat Genet 2015;47:1121-30.

DOI: 10.1056/NEJMc1607380

TO THE EDITOR: In the studies reported by Dewey et al. and Stitziel et al., the reduced risk of cardiovascular disease observed among carriers of inactivating variants in ANGPTL4 suggests a causal role for plasma triglycerides. However, in both studies, lower triglyceride levels in carriers were inextricably linked with elevated levels of highdensity lipoprotein (HDL) cholesterol. In another 
genetic study that decoupled HDL cholesterol levels from triglyceride levels, there was no association between HDL cholesterol levels and the risk of cardiovascular disease. ${ }^{1}$ However, similar data from a study that disentangles triglyceride levels from HDL cholesterol levels are still lacking.

Inactivating $A P O C 3$ variants have been associated with reduced triglyceride levels, elevated HDL cholesterol levels, and a reduced risk of cardiovascular disease, ${ }^{2}$ whereas inactivating APOA5 variants have been associated with elevated triglyceride levels, reduced HDL cholesterol levels, and an elevated risk of cardiovascular disease. ${ }^{3}$ Modulation of lipoprotein lipase activity might be a unifying explanation. Indeed, LPL variants that influence triglyceride and HDL cholesterol levels reciprocally are also associated with the risk of cardiovascular disease. However, unduly emphasizing the triglyceride level as causal disregards a hard-earned biologic understanding that lipolysis affects HDL cholesterol levels concurrently. In this situation, it may be premature to overlook HDL cholesterol. The joint triglycerideHDL cholesterol phenotype may mark causal processes that are otherwise inaccessible to measurement in epidemiologic-scale samples.

Robert A. Hegele, M.D.

Robarts Research Institute

London, ON, Canada

hegele@robarts.ca

No potential conflict of interest relevant to this letter was reported.

1. Voight BF, Peloso GM, Orho-Melander M, et al. Plasma HDL cholesterol and risk of myocardial infarction: a mendelian randomisation study. Lancet 2012;380:572-80.

2. Jørgensen AB, Frikke-Schmidt R, Nordestgaard BG, TybjærgHansen A. Loss-of-function mutations in APOC3 and risk of ischemic vascular disease. N Engl J Med 2014;371:32-41.

3. Do R, Stitziel NO, Won HH, et al. Exome sequencing identifies rare LDLR and APOA5 alleles conferring risk for myocardial infarction. Nature 2015;518:102-6.

DOI: 10.1056/NEJMc1607380

TO THE EDITOR: Dewey et al. and Stitziel et al. report that reduced triglyceride levels in carriers of loss-of-function variants in ANGPTL4 were associated with a reduced cardiovascular risk. Lower risk was attributed to lower levels of triglycerides in carriers than in noncarriers, whereas levels of low-density lipoprotein (LDL) cholesterol were similar and normal in carriers and noncarriers.

Neither study included assays of apolipoprotein B (apoB), the sum of very-low-density lipoprotein (VLDL) and LDL particle numbers. Even in patients with hypertriglyceridemia, apoB LDL particles compose more than $85 \%$ of the total number of apoB-lipoprotein particles. If the apoB level is elevated, increased numbers of cholesteroldepleted LDL particles contribute to an increased cardiovascular risk. ${ }^{1}$

The findings in Justification for the Use of Statins in Prevention: an Intervention Trial Evaluating Rosuvastatin (JUPITER) ${ }^{2}$ are pertinent. JUPITER was a primary prevention trial of statin therapy involving healthy persons with low levels of LDL cholesterol but high levels of C-reactive protein. Triglyceride levels were similar to those in the studies by Dewey et al. and Stitziel et al., but apoB, and therefore the LDL particle number, was elevated. ${ }^{2}$ JUPITER showed that statins lowered apoB levels and rates of clinical events, but it did not provide evidence that lowering levels of triglycerides is clinically beneficial.

Accordingly, we think that knowing the levels of apoB would enhance our understanding of the results of these two studies. Normal levels of apoB in persons at risk would implicate triglycerides, whereas elevated levels of apoB would implicate LDL particles.

Allan D. Sniderman, M.D.

George Thanassoulis, M.D.

McGill University Health Centre

Montreal, QC, Canada

allansniderman@hotmail.com

Dr. Thanassoulis reports receiving lecture fees from Servier Canada, fees for serving on advisory boards for Servier Canada, Amgen, and Ionis Pharmaceuticals, and consulting fees from Ionis Pharmaceuticals. No other potential conflict of interest relevant to this letter was reported.

1. de Graaf J, Couture P, Sniderman AD. ApoB in clinical care. Houten, the Netherlands: Springer Media BV, 2015.

2. Ridker PM, Danielson E, Fonseca FAH, et al. Reduction in C-reactive protein and LDL cholesterol and cardiovascular event rates after initiation of rosuvastatin: a prospective study of the JUPITER trial. Lancet 2009;373:1175-82.

DOI: $10.1056 /$ NEJMc1607380

TO THE EDITOR: Stitziel et al. report that a common gain-of-function genetic variant in LPL, which encodes lipoprotein lipase, is associated with a reduction in the risk of coronary artery disease. The authors speculate that therapeutic activation of this pathway might reduce the risk of coronary artery disease.

To test this hypothesis, we analyzed data from 4414 persons involved in the ACCORD Lipid randomized clinical trial ${ }^{1,2}$ to determine whether the cardioprotective effect of fenofibrate (a drug with widespread effects on lipid metabolism, including LPL activation) ${ }^{3}$ was influ- 


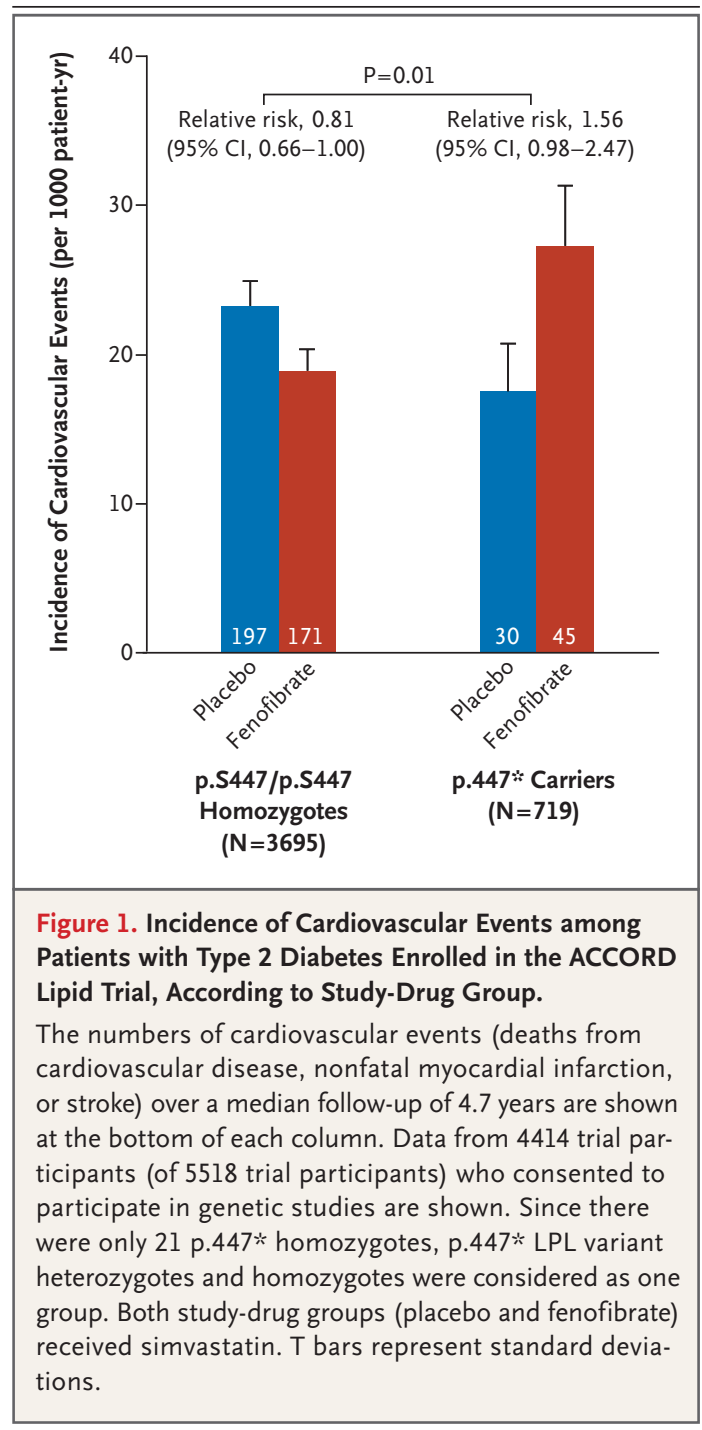

enced by the common gain-of-function LPL variant (p.S447^) described in the article by Stitziel et al. In the ACCORD Lipid trial, patients with type 2 diabetes who were receiving simvastatin and who were at high risk for cardiovascular events were assigned to receive either fenofibrate or placebo. In the overall ACCORD Lipid trial, fenofibrate did not have a significant effect on cardiovascular events. ${ }^{1}$ However, consistent with the working hypothesis, a negative interaction $(\mathrm{P}=0.01)$ was observed between the use of fenofibrate and p.S447*. Fenofibrate was beneficial in p.S447 homozygotes but not in carriers of the gain-of-function p.447^ variant (Fig. 1). By showing that there was no association between fenofibrate and protection against risk among persons in whom LPL activity was already elevated, our findings provide some support for the importance of this pathway as a target for cardioprotective interventions.

Mario L. Morieri, M.D.

Hetal Shah, M.D., M.P.H.

Alessandro Doria, M.D., M.P.H.

Joslin Diabetes Center

Boston, MA

alessandro.doria@joslin.harvard.edu

for the Action to Control Cardiovascular Risk in Diabetes (ACCORD) Genetic Study Group

No potential conflict of interest relevant to this letter was reported.

1. Ginsberg HN, Elam MB, Lovato LC, et al. Effects of combination lipid therapy in type 2 diabetes mellitus. $N$ Engl J Med 2010;362:1563-74.

2. Shah HS, Gao H, Morieri ML, et al. Genetic predictors of cardiovascular mortality during intensive glycemic control in type 2 diabetes: findings from the ACCORD clinical trial. Diabetes Care 2016;39:1915-24.

3. Duval C, Müller M, Kersten S. PPARalpha and dyslipidemia. Biochim Biophys Acta 2007;1771:961-71.

DOI: 10.1056/NEJMc1607380

DR. DEWEY AND COLLEAGUES REPLY: We welcome the report that Morrison et al. have replicated our finding of reduced odds of coronary artery disease in persons carrying the ANGPTL4 p.E40K variant, as well as the report that Morieri et al. have observed an interaction between the LPL genotype and fenofibrate with respect to rates of cardiovascular events.

We agree with Hegele that the joint triglyceride-HDL cholesterol phenotype that is associated with the variant ANGPTL4 complicates the interpretation of the mechanistic link between the triglyceride level and the risk of coronary artery disease. As discussed in our article, we could not rule out an effect of variant ANGPTL4 through alteration of HDL cholesterol levels on the risk of disease, although a genetic study suggests that certain mechanisms of alteration of HDL cholesterol levels are not linked with the risk of coronary artery disease. ${ }^{1}$ The total nonHDL-cholesterol fraction, which includes the apoB-containing triglyceride-rich VLDL and intermediate-density lipoproteins, as well as chylomicrons and their remnants, have also been linked epidemiologically ${ }^{2,3}$ and now genetically ${ }^{4}$ to the risk of coronary artery disease and may contribute to the associations observed in our study. It is not yet clear which changes in lipid fractions causally mediate the reduction in atherosclerotic coronary artery disease.

We agree with Sniderman and Thanassoulis 
that assays of apoB levels in persons with variants in ANGPTL4 and in those without these variants would shed further light on the connection between the lipid associations observed in our study and the risk of coronary artery disease. Indeed, Desai et al. ${ }^{5}$ found that VLDL cholesterol clearance is increased and VLDL cholesterol production is decreased in Angptl4-deficient mice as well as in mice treated with a blocking antibody to ANGPTL4. This finding suggests that this apoB-containing triglyceride-rich lipoprotein fraction is modulated by ANGPTL4 activity. We did not assay apoB levels in study participants and agree that it would be helpful to do so in future studies involving carriers of variant ANGPTL4.

Frederick E. Dewey, M.D.

Jesper Gromada, Ph.D., D.M.Sc.

Alan R. Shuldiner, M.D.

Regeneron Genetics Center

Tarrytown, NY

frederick.dewey@regeneron.com

Since publication of their article, the authors report no further potential conflict of interest.

1. Voight BF, Peloso GM, Orho-Melander M, et al. Plasma HDL cholesterol and risk of myocardial infarction: a mendelian randomisation study. Lancet 2012;380:572-80.

2. Rana JS, Boekholdt SM, Kastelein JJ, Shah PK. The role of non-HDL cholesterol in risk stratification for coronary artery disease. Curr Atheroscler Rep 2012;14:130-4.

3. Di Angelantonio E, Sarwar N, Perry P, et al. Major lipids, apolipoproteins, and risk of vascular disease. JAMA 2009;302: 1993-2000.

4. Helgadottir A, Gretarsdottir S, Thorleifsson G, et al. Variants with large effects on blood lipids and the role of cholesterol and triglycerides in coronary disease. Nat Genet 2016;48:634-9. 5. Desai U, Lee EC, Chung K, et al. Lipid-lowering effects of antiangiopoietin-like 4 antibody recapitulate the lipid phenotype found in angiopoietin-like 4 knockout mice. Proc Natl Acad Sci U S A 2007;104:11766-71.

DOI: 10.1056/NEJMc1607380

DR. STITZIEL REPLIES: My colleagues and I are pleased to see that Morrison and colleagues have replicated the association between ANGPTL4 p.E40K and a reduced risk of coronary artery disease.

Rare mutations within several genes - APOA,${ }^{1}$
APOC $3,2,3$ and now ANGPTL4 - are associated with the risk of coronary artery disease as well as plasma concentrations of triglyceride-rich lipoproteins and HDL. The product of each of these genes regulates the activity of lipoprotein lipase anchored to the luminal surface of endothelial cells. As such, Hegele suggests that enzymatic activity of lipoprotein lipase along with its reciprocal effects on triglyceride-rich lipoproteins and HDL may be a pathophysiologic link to coronary artery disease. We agree that a combined phenotype of triglyceride-rich lipoproteins and HDL may prove to be useful as an indirect biomarker of lipoprotein lipase activity.

Sniderman and Thanassoulis ask for data on apoB particles. This is clearly of interest, but apoB levels were not assayed in the majority of patients in our study.

Morieri and colleagues report a pharmacogenetic interaction between a gain-of-function variant in the lipoprotein lipase gene (p.S447`) and the efficacy of fenofibrate therapy. This interesting finding, which requires replication, may hold potential for personalized therapy with this class of drugs in patients with diabetes.

Nathan O. Stitziel, M.D., Ph.D.

Washington University School of Medicine

St. Louis, MO

nstitziel@wustl.edu

\section{for the Myocardial Infarction Genetics} and CARDIoGRAM Exome Consortia Investigators

Since publication of his article, the author reports no further potential conflict of interest.

1. Do R, Stitziel NO, Won HH, et al. Exome sequencing identifies rare LDLR and APOA5 alleles conferring risk for myocardial infarction. Nature 2015;518:102-6.

2. The TG and HDL Working Group of the Exome Sequencing Project, National Heart, Lung, and Blood Institute. Loss-of-function mutations in $A P O C 3$, triglycerides, and coronary disease. N Engl J Med 2014;371:22-31.

3. Jorgensen AB, Frikke-Schmidt R, Nordestgaard BG, TybjærgHansen A. Loss-of-function mutations in APOC 3 and risk of ischemic vascular disease. N Engl J Med 2014;371:32-41.

DOI: $10.1056 /$ NEJMc1607380

\section{Biologic and Clinical Perspectives on Thyroid Cancer}

TO THE EDITOR: In their review article on thyroid cancer, Fagin and Wells (Sept. 15 issue) ${ }^{1}$ state that $7 \%$ of unselected medullary thyroid carcinomas are attributable to germline RET mutations, but they do not provide a similar numerical esti- mate of genes that contribute to the development of differentiated thyroid carcinoma. This lack of an estimate and the authors' mention of PTEN, $A P C$, and WRN as susceptibility genes for differentiated thyroid carcinoma prompted us to ana- 